\title{
Should the GCM2 gene be tested when screening for familial primary hyperparathyroidism?
}

\author{
Lucie Coppin ${ }^{1,2}$, Margaux Dufosse ${ }^{2}$, Pauline Romanet ${ }^{3}$, Sophie Giraud4, Marie-Odile North5, \\ Catherine Cardot Bauters ${ }^{6}$, Françoise Borson-Chazot ${ }^{7}$, Laurence Duchesne ${ }^{8}$, Mélanie Métallo9, \\ Tonio Lovecchio², Anne Barlier ${ }^{3}$ and Marie-Françoise Odou²,10
}

${ }^{1}$ Universite de Lille, Inserm, CHU Lille, UMR-S1172-JPArc, Centre de Recherche Jean Pierre Aubert Neurosciences et Cancer, Lille Cedex, France, ${ }^{2}$ Service de Biochimie et Biologie Moléculaire 'Hormonologie, Métabolisme-Nutrition, Oncologie', CHU Lille, Lille, France, ${ }^{3}$ Aix Marseille Université, APHM, INSERM, MMG, Laboratory of Molecular Biology Hospital La Conception, Marseille, France, ${ }^{4}$ Genetics Department, Hospices Civils de LYON (HCL), University Hospital, East Pathology Center, Bron Cedex, France, ${ }^{5}$ Service de Génétique et Biologie Moléculaires, Hôpital Cochin, Assistance Publique - Hôpitaux de Paris, Paris, France, ${ }^{6}$ Service d'Endocrinologie, Hôpital Claude Huriez, CHU Lille, Lille Cedex, France, ${ }^{7}$ Hospices Civils de Lyon, Fédération d'Endocrinologie, Université Claude Bernard Lyon 1, Lyon, France, ${ }^{8}$ Service d'Endocrinologie, CHR Mercy, Metz, France, ${ }^{9}$ Department of Endocrinology, University Hospital Center of Nancy, Nancy, France, and ${ }^{10}$ Faculty of Pharmacy, UMR995, LIRIC (Lille Inflammation Research International Center), University of Lille, Lille, France

Correspondence should be addressed to M-F Odou Email marie-francoise.odou@ chru-lille.fr

\begin{abstract}
Objective: Primary hyperparathyroism (PHPT) is a disease with either sporadic or inherited presentation. Germline mutations responsible for this disease can be found in different genes, the most frequently involved being MEN1, CDC73 = HRPT2 and CASR. During the last few years, new genes have been described as responsible for the development of PHPT such as GCM2. These genes are not systematically included in PHPT genetic screening yet. The aim of this work was to assess the importance of GCM2 genetic analysis in PHPT to determine if this gene should be included in gene panel investigated for this disease.

Design and methods: The TENGEN network (French Oncogenetic Network of Neuroendocrine Tumors) collected and interpreted allelic variants according to the clinical characteristics of the GCM2-positive patients identified through genetic testing performed in French laboratories (713 patients with PHPT).

Results: From 713 patients with PHPT included in this study, 85 (6.6\%) carried at least one GCM2 variant. A total of 12 variants classified as uncertain significance or likely pathogenic were reported in 47 patients. Their mean age at PHPT diagnosis was 49 years. Additionally, the investigation of a large family showed that GCM2 variants could be associated with low penetrance.

Conclusion: We provide a description and interpretation for GCM2 variants identified in a French population. We suggest that this gene should be included in genetic screening of patients with PHPT and propose the follow-up of asymptomatic patients carrying such variants for calcemia.
\end{abstract}

European Journal of Endocrinology

(2020) 182, 57-65

\section{Introduction}

Primary hyperparathyroidism (PHPT) is a disorder characterized by hypercalcemia and elevated or inappropriate parathyroid hormone (PTH) secretion due to pararathyroid gland hyper-functioning. It can result from a single or multiglandular lesion, adenoma, hyperplasia or much less frequently carcinoma. The prevalence of this https://eje.bioscientifica.com https://doi.org/10.1530/EJE-19-0641
(C) 2020 European Society of Endocrinology Printed in Great Britain
Published by Bioscientifica Ltd. 
disease is estimated around 1-7 cases per 1000 persons (1). PHPT can be either sporadic or inherited. Among inherited PHPT (5-10\% of cases), some belong to syndromic diseases such as multiple endocrine neoplasia type 1 (MEN1; due to mutations in MEN1 tumor suppressor gene), multiple endocrine neoplasia type 2A (MEN2A; due to mutations in RET proto-oncogene), multiple endocrine neoplasia type 4 (MEN4; due to mutations in CDKN1B proto-oncogene) or hyperparathyroidism-jaw tumor syndrome (HPT-JT; due to mutation in $C D C 73=H R P T 2$ ). PHPT can also be non-syndromic and isolated. The cases of familial isolated PHPT (FIHP) can be included in incomplete expression of MEN1, MEN4, HPT-JT or associated with mutation in CASR, GNA11 or AP2S1 genes $(2,3)$. In recent years, activating mutations in GCM2, a gene previously known to be involved in inherited hypoparathyroidism, have been described in PHPT $(4,5)$. Cases of PHPT associated with GCM2 mutations in literature are characterized by multiple enlarged parathyroid glands, greater preoperative parathyroid hormone levels than patients without GCM2 mutations, persistent or recurrent PHPT after surgery and higher risk of parathyroid carcinoma (6). Currently, frequency of GCM2 mutation in FIHP is estimated to be around $18 \%$ in literature (5).

GCM2 is located in locus 6p34. It encodes for a 506 amino acid protein composed of several functional domains: a DNA-binding domain, 2 transactivation domains, a C-terminal conserved inhibitory domain (CCID) and a nuclear localization signal. This protein, which is exclusively expressed in parathyroid cells, acts as a transcription factor involved in parathyroid gland differentiation and development and in PTH production. Knockout mice for GCM2 are characterized by parathyroid agenesis and hypoparathyroidism (7). GCM2-inactivating mutations have been involved in hypoparathyroidism and more recently, activating germline mutations in this gene have been described in association with hyperparathyroidism, suggesting that GCM2 is a parathyroid proto-oncogene. These gain-of-function mutations are mostly located in the CCID domain (5).

Currently, genetic screening recommendation for PHPT in France do not include GCM2 analysis. The aim of our study was to investigate germline GCM2 sequence variations in a French cohort of patients with PHPT and to classify the identified variants according to ACMGAMP guidelines $(8,9)$ in order to determine if this gene should be systematically included in PHPT gene screening decision tree. The deleterious effects of some sequence variants are controversial in literature and are still open for debate.

\section{Material and methods}

\section{GCM2 sequencing}

Patients selected in this study between 2015 and 2018 presented with a familial history of PHPT or exhibited a sporadic presentation before the age of 50 years, recurrent/ persistent PHPT or multiple gland parathyroid lesions, according to French guidelines of genetic screening for PHPT (available at http://www.reseau-gte.org/). They all signed an informed consent allowing germline DNA analysis during a one-on-one genetic counseling. The study was validated and approved by Ethical Committee (Comité de Protection des Personnes (CPP Nord-Ouest IV)) under number HP 1930. In total, 713 independent probands were included in the study. Blood samples were addressed to one of the four French laboratories from the TENGEN network (French oncogenetic network on neuroendocrine tumors) analyzing GCM2.

DNA was extracted from leucocytes and sequenced by targeted next-generation sequencing. Sequence variations in GCM2 exons and exon-intron boundaries were all confirmed by Sanger sequencing (primer sequences available on demand).

Additionally, 13 relatives from a family, presenting or not with primary hyperparathyroidism, were screened on two independent blood samples for a GCM2-specific variant by targeted Sanger sequencing. They also all signed an informed consent.

\section{Classification of GCM2 variants}

Variants are written according to HGVS nomenclature (with NM_004752.3, assembly GRCh37/hg19 version of human genome reference).

Sequence variants in GCM2 were interpreted and classified according to ACMG-AMP guidelines taking into account open access population database frequencies (dbSNP, gnomAD, ESP, ExAc), in silico prediction softwares, the available familial, clinical and biological data and scientific publications. Classification for each variant in one out of the five classes was validated by the French TENGEN expert group (class 1: benign; class 2: likely benign; class 3: uncertain significance; class 4: likely pathogenic; class 5: pathogenic).

\section{Results}

Seven hundred thirteen patients with PHPT were included in this study. Excluding a very frequent variant identified 
in about $10 \%$ of the DNA samples and directly considered as a benign class 1 variant (c.-44T>C, rs16870746, $10.73 \%$ in gnomAD(2.1)), 85 patients carried at least one GCM2 variant.

To be more accurate, we identified 12 variants classified as class 1 or 2 variants (benign or likely benign) according to ACMG criteria (Table 1). Interestingly, some of these variants were cosegregating variants such as c.679A $>\mathrm{G}$, p.(Ile227Val) associated with c.350C $>$ T, p.(Ala117Val) and c.462G >A, p.(Lys154Lys) associated with c.607G>A, p.(Gly203Ser). These class 1 or 2 variants were not taken into account for genotype-phenotype correlation, as they were very frequent and considered as SNP.

Twelve sequence variations were classified as class 3 (uncertain significance) or 4 (likely pathogenic) variants (Table 2). No sequence variation was classified in class 5 (pathogenic). Forty-seven index cases harbored these classes of variants, which correspond to a frequency of 6.6\% (47/713). More accurately, 44 patients were carriers of a class 3 variant and three patients carried a class 4 variant. Interestingly, all these sequence variations were substitutions. We identified no deletion or duplication in these classes of variants. Main clinical characteristics of the corresponding patients are presented in Table 3. For these patients, mean age of PHPT diagnosis was 49 years (min: 13; max: 82). Nine patients harbored parathyroid hyperplasia, 17 patients presented with parathyroid adenoma and anatomopathological data were not available for the other patients.

In parallel of the study of this cohort, we had the opportunity to perform genetic investigations in a family (genealogic tree in Fig. 1). The index case, a 28-year-old man, presented with a $20 \mathrm{~mm}$ parathyroid adenoma associated with a calcemia level of $4.15 \mathrm{mmol} / \mathrm{L}$ and a
PTH level of $355 \mathrm{pg} / \mathrm{mL}$. Our genetic analyses allowed to identify the c.1181A>C, p.(Tyr394Ser) class 4 variant. Interestingly, above the 13 relatives analyzed in this family, we identified this variant in 7 of them: four members carrying this variant at heterozygous state but without PHPT respectively at the age of $80(\mathrm{Ca}: 2.4 \mathrm{mmol} / \mathrm{L}$ and PTH: $30.2 \mathrm{pg} / \mathrm{mL}$ ), 58 (Ca: $2.3 \mathrm{mmol} / \mathrm{L}$ and PTH: $6 \mathrm{pg} /$ $\mathrm{mL}), 34$ and 33. The three other members of this family harboring the variant had a symptomatic PHPT.

\section{Discussion}

In our study, 47 patients out of 713 were carriers of a class 3 or class 4 variant, none of them carried a class 5 variant. This represents $6.6 \%$ of the patients screened for PHPT in our population. Guan et al. (5), who selected kindreds with FIHP, found a frequency of $18 \%$ of patients with a GCM2 variant and without deleterious mutation on the current other genes involved in heritable hyperparathyroidism. This observed discrepancy can be due to selection of familial cases in their study and to prior exclusion of mutation in MEN1, $C A S R$, and CDC73, while our cohort included all patients screened for heritable PHPT, including sporadic cases. Sporadic presentation in case of genetically determined PHPT is not so rare because PHPT can be asymptomatic over many years, knowledge of first- but especially secondor third-degree relative affected with PHPT is not always obvious, and incomplete penetrance of pathology with genetic abnormality is also to be considered. That is the reason why genetic screening is recommended in France for all patients of less than 50 years with PHPT, even with sporadic presentation. The mean age of PHPT diagnosis in patients carriers of a class 3 or 4 GCM2 variant in our

Table 1 Classes 1 and 2 variants. Frequencies in African population and results of functional evaluations are indicated when available and informative for interpretation.

\begin{tabular}{|c|c|}
\hline Nucleotide variation & Protein variation \\
\hline C. $-44 \mathrm{~T}>\mathrm{C}$ & p.(?) \\
\hline c.344-7T>C & p.(?) \\
\hline c. $350 C>T$ & p.(Ala117Val) \\
\hline c. $462 \mathrm{G}>\mathrm{A}$ & p.(Lys154Lys) \\
\hline c. $607 \mathrm{G}>\mathrm{A}$ & p.(Gly203Ser) \\
\hline c. $679 A>G$ & p.(Ile227Val) \\
\hline c. $739 \mathrm{C}>\mathrm{T}$ & p.(Leu247Leu) \\
\hline c.897T >C & p.(Pro299Pro) \\
\hline c. $903 \mathrm{~T}>\mathrm{C}$ & p.(Asp301Asp) \\
\hline c. $1060 A>G$ & p.(Met354Val) \\
\hline c.1177_1185dup & p.(Ala393_Gln395dup) \\
\hline c. $1395 \mathrm{C}>\mathrm{T}$ & p.(His465His) \\
\hline
\end{tabular}

\begin{tabular}{c}
\hline Frequency in gnomAD $(\mathrm{v} 2.1)$ (All; \%) \\
\hline 10.73 \\
0.13 \\
0.11 (AFR: $1.2 \%)$ \\
1.0 (AFR: $10.28 \%)$ \\
1.0 (AFR: $10.26 \%)$ \\
0.25 (AFR: $2.7 \%)$ \\
0.002 \\
0.87 (AFR: $5.06 \%)$ \\
0.86 \\
0.41 (AFR: $4.16 \%)$ \\
0.13 \\
0.027
\end{tabular}

Results of functional studies

NS, no significant effect on transcription in comparison with wild-type sequence. 
Table 2 Classes 3 and 4 variants.

\begin{tabular}{|c|c|c|}
\hline $\begin{array}{l}\text { Nucleotide } \\
\text { variation }\end{array}$ & Protein variation & ACMG class proposition \\
\hline c. $-13 G>A$ & p.(?) & 3 \\
\hline c.35T >A & p.(Val12Glu) & 3 \\
\hline c. $139 \mathrm{C}>\mathrm{T}$ & p.(Arg47Cys) & 3 \\
\hline c. $157 \mathrm{G}>\mathrm{A}$ & p.(Asp53Asn) & 3 \\
\hline c. $168 \mathrm{G}>\mathrm{C}$ & p.(Lys56Asn) & 3 \\
\hline c.319G $>A$ & p.(Asp107Asn) & 3 \\
\hline c.809G $>A$ & p.(Arg270Lys) & 3 \\
\hline $\mathrm{c.844T}>\mathrm{G}$ & p.(Tyr282Asp) & 3 \\
\hline c. $943 \mathrm{~A}>\mathrm{G}$ & p.(Asn315Asp) & 3 \\
\hline c.989A $>\mathrm{T}$ & p.(Gln330Leu) & 3 \\
\hline C. $1181 \mathrm{~A}>\mathrm{C}$ & p.(Tyr394Ser) & 4 \\
\hline c. $1217 \mathrm{G}>\mathrm{A}$ & p.(Arg406Gln) & 3 \\
\hline
\end{tabular}

study was 49 years, which stands between mean age of parathyroidectomy of family presenting PHPT patients (42.8 years) and sporadic presenting PHPT patients (59.2 years) in the study of El Lakis et al. (1).

Castellano et al. (10) reported a female-to-male ratio of 3:1 in PHPT patients. In our study, this ratio was 2:1 in patient carriers of a class 3 or 4 GCM2 variant. This more equilibrated female-to-male ratio is consistent with a genetic predisposition to the pathology and could thus be explained by the Mendelian transmission.

El Lakis et al. (1) also demonstrated that activating mutations of GCM2 were associated with a greater rate of multi-glandular diseases. In our study, some patients with class 3 or 4 variants harbored parathyroid hyperplasia or multiple glandular adenomas but the lack of anatomopathological information available for many patients did not allow us to confirm this point.

A great number of variants are still classified as class 3 variants (variant of uncertain significance). Indeed, even when in vitro functional data are available, their interpretation can remain tricky, as for the c.844T>G, p.(Tyr282Asp) variant. In our cohort, this variant has been identified in association with a deleterious variant once in MEN1 gene and once in CASR gene. As a transcription factor expressed in parathyroid cells and activating PTH transcription, GCM2 can contribute to development of PHPT but, may be, to a lesser extent than MEN1, CDKN1B or HRPT2 (11). Interaction with CASR expression in parathyroid cells has been described, as well as interaction with GATA3 and MafB for PTH expression, but some compensation mechanisms seem to exist $(12,13)$. This could explain the low penetrance observed, as shown with our family study.

The pathogenicity of p.(Tyr282Asp) variant is controversial in literature and its role in tumorigenesis is not proven (14). D'Agruma et al. (4) demonstrated that this variant was associated with hypercalcemia and exhibited increased transcriptional activity compared to the wild-type protein. In the same way, Marchiori et al. (15) showed that this variant was associated with increased serum Ca levels. On the contrary, Guan et al. (5) demonstrated that the transcriptional activity of this variant was similar to the wild-type sequence. This variant is not localized in the CCID domain where activating mutations have mostly been identified. The mutated position is not well conserved among species and in silico predictions mainly predict the variant as benign (six prediction software considered it as benign vs three pathogenic). Frequency of p.(Tyr282Asp) is currently of $1.23 \%$ in ESP database (European American). This frequency over $1 \%$ in population could tilt the balance in favor of the interpretation of this variant as a benign polymorphism, even if the threshold of 5\% is not reached, which would constitute a stand-alone evidence of benign impact according to ACMG-AMP guidelines. Anyway, an enrichment of this sequence variation is noticed in patients with PHPT, as initially described by D'Agruma et al. (4). It is also the case in our cohort in comparison with the control population of gnomAD v2.1 database (30/1426 VS. 2549/282896, OR 2.3 (IC95: 1.6 to 3.4)), but too moderately to be considered as an argument for a pathogenic effect. Moreover, in our cohort, this variant has been identified in two patients with pathogenic variation in another gene: one patient had a pathogenic inactivating substitution in CASR (NM_000388.3 c.164C $>$ T) and the other patient had a deletion in MEN1 (NM_130799.2 c.776del). Finally, the selected criteria for ACMG classification could be as follows: PS4 (The prevalence of the variant in affected individuals is significantly increased compared with the prevalence in 


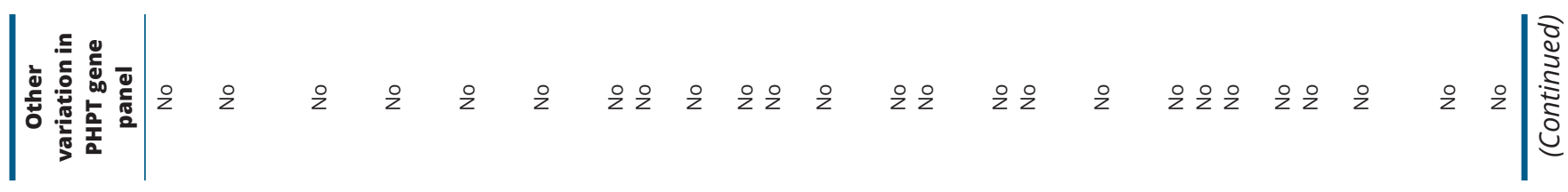

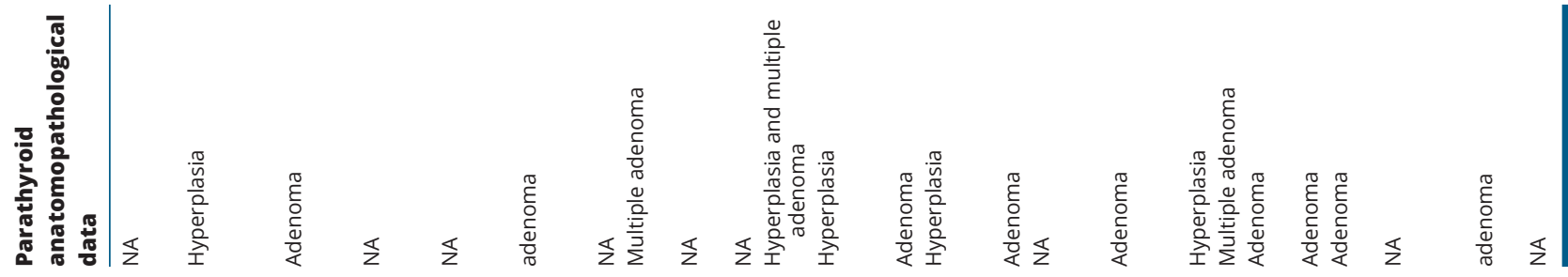
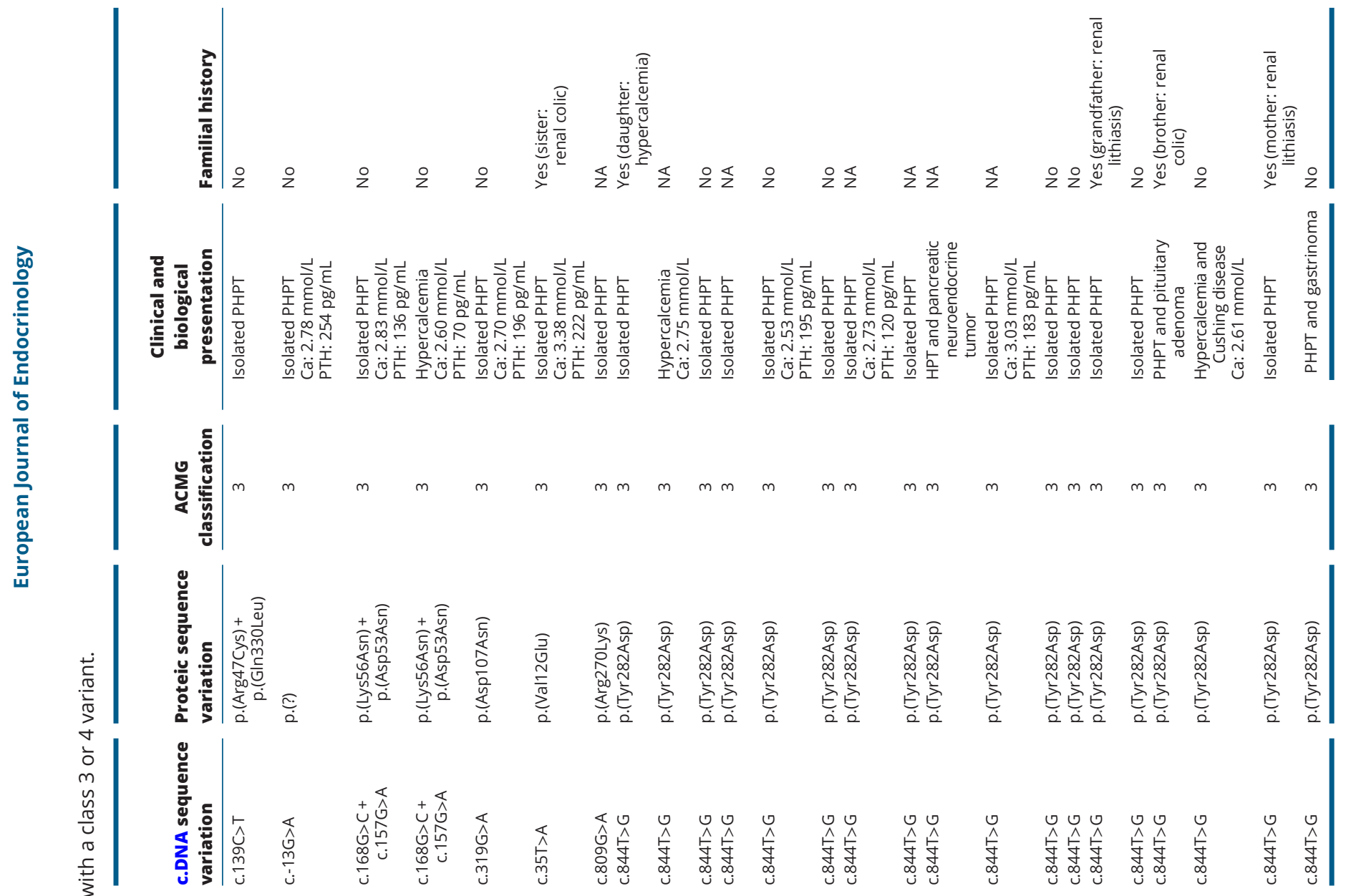


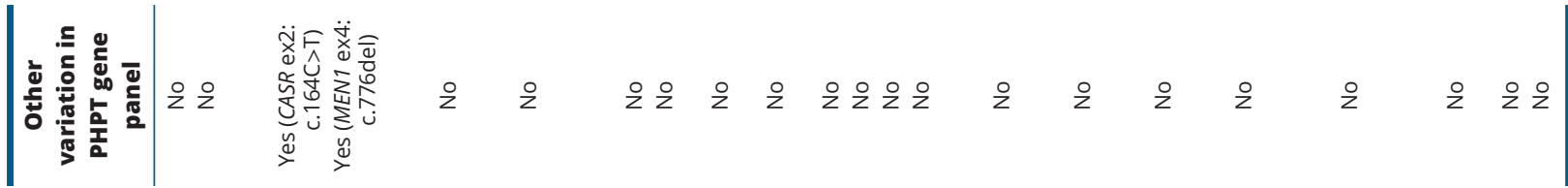
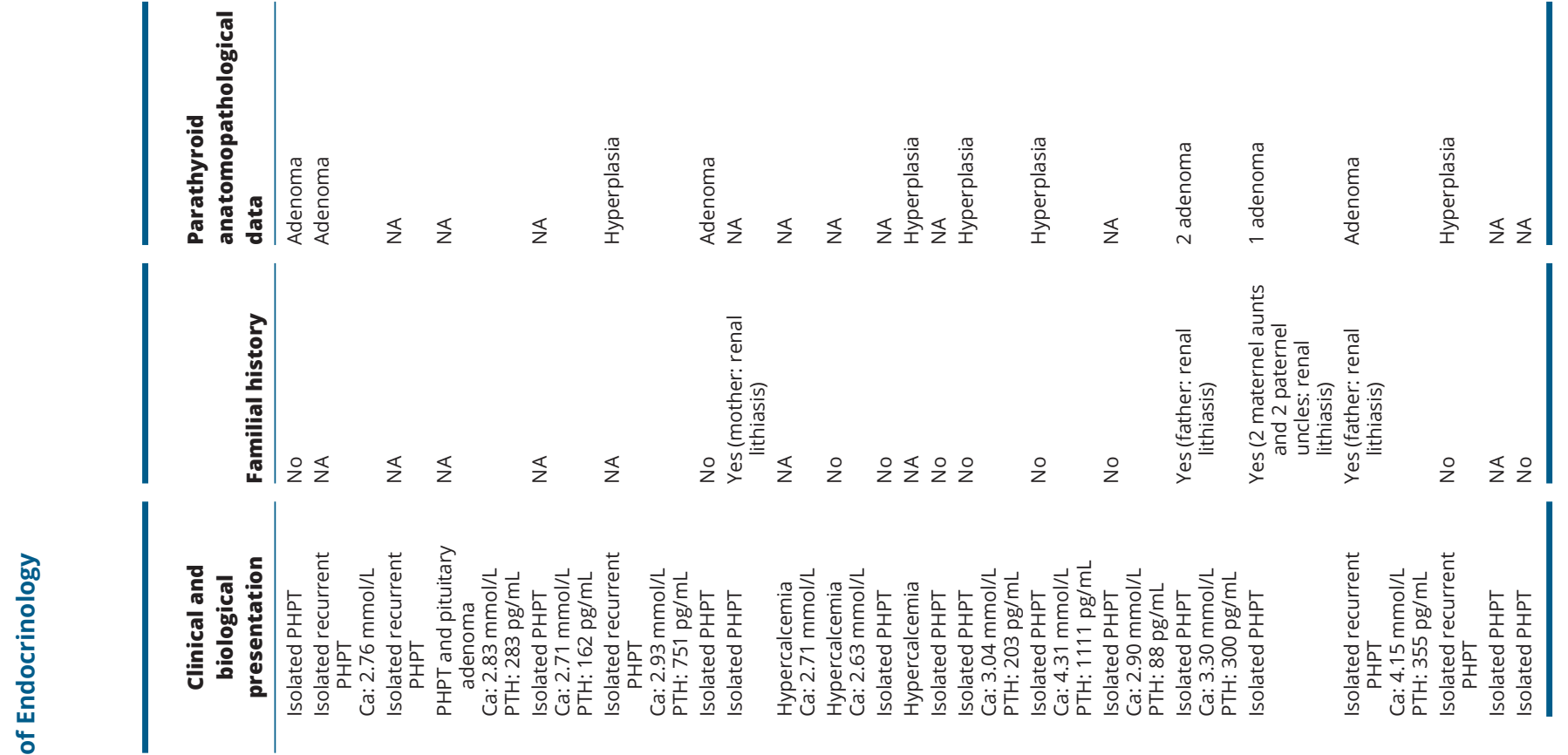

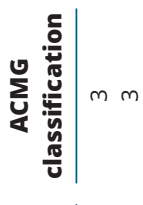

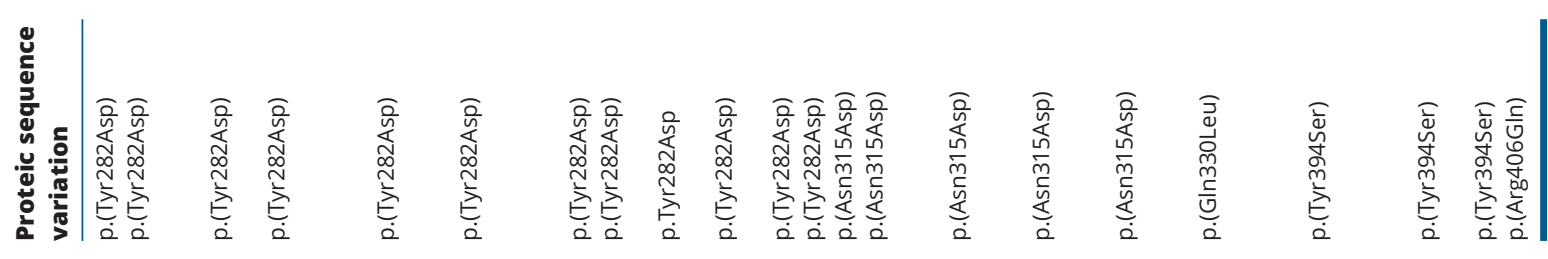

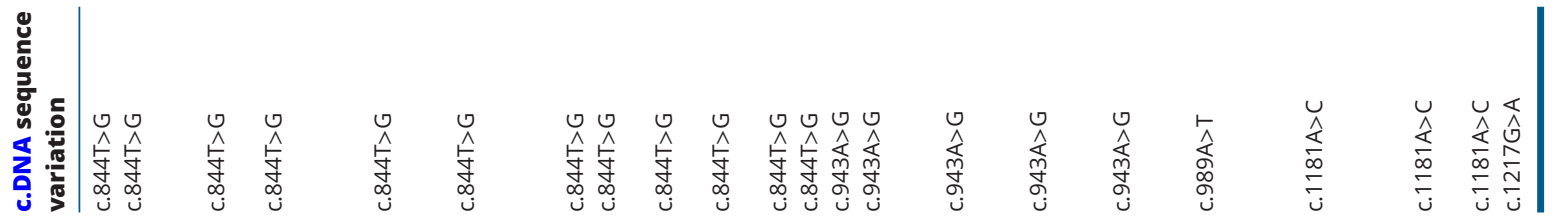

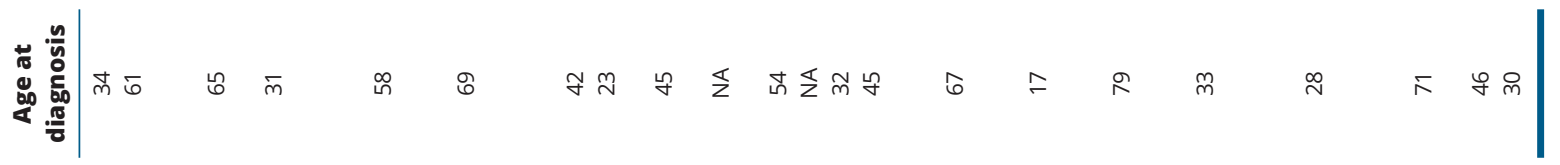

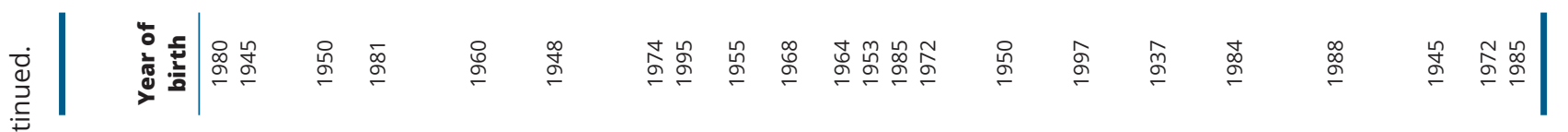

它|

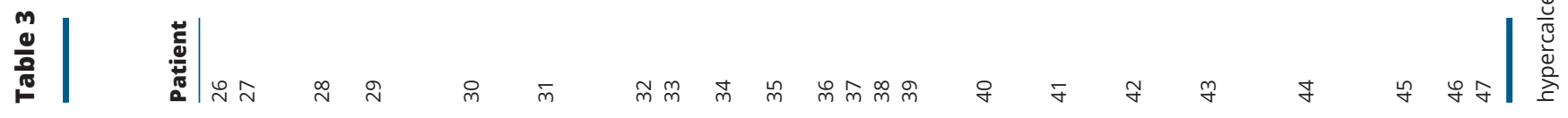




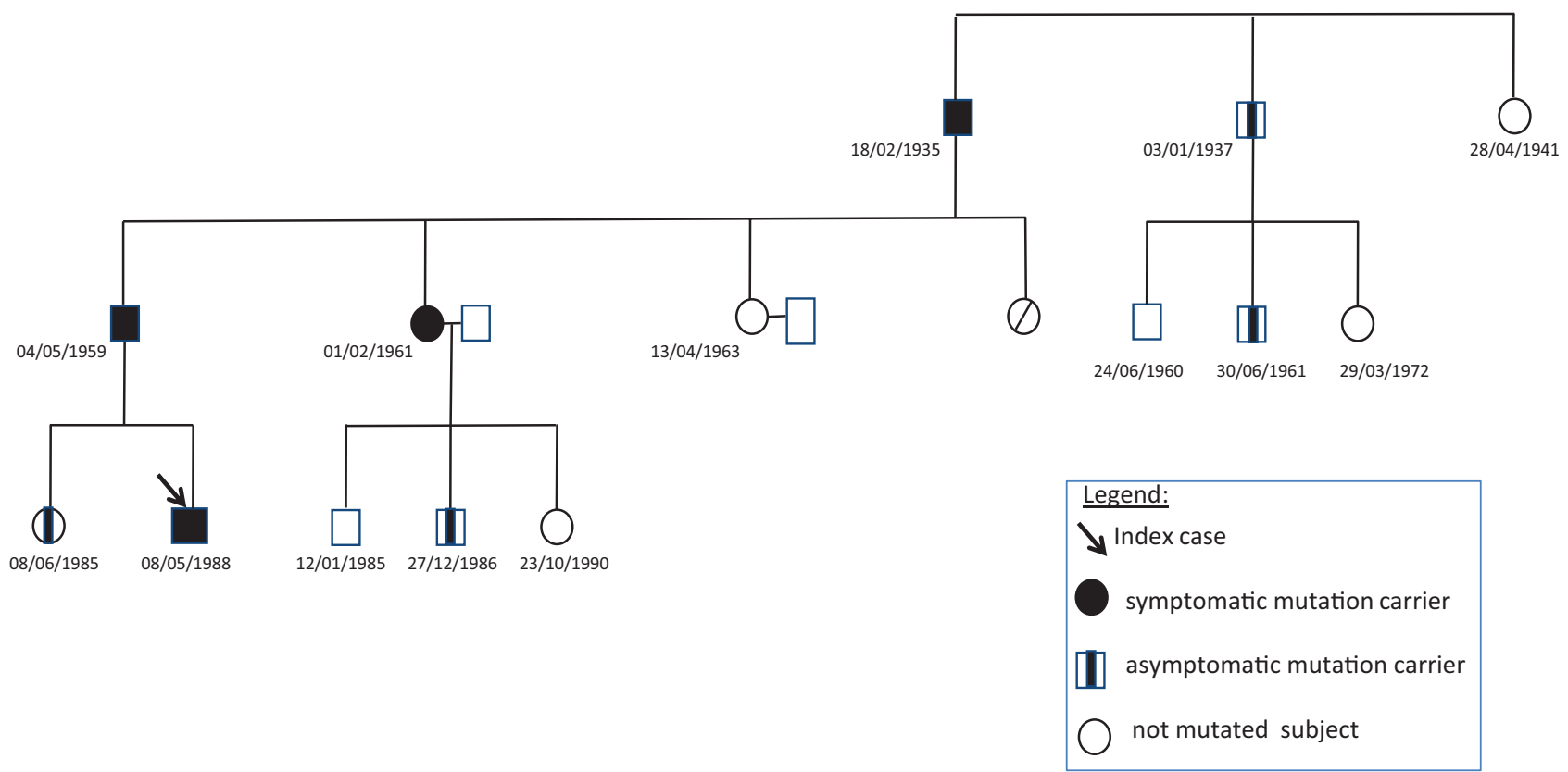

Figure 1

Genealogic tree of the family with GCM2 (NM_004752.3) c.1181A>C, p.(Tyr394Ser) variant.

controls) + BP4 (Multiple lines of computational evidence suggest no impact on gene or gene product (conservation, evolutionary, splicing impact, etc...)) resulting in classification as a class 3 variant, that is variant of uncertain significance. PP5 criteria (Reputable source recently reports variant as pathogenic, but the evidence is not available to the laboratory to perform an independent evaluation) was not applied because the functional effect of this variant is controversial in literature. All these elements could suggest that this sequence variation is a phenotype modifier variant, as it has been described for the frequent variant p.(Ala986Ser) in CASR, described as contributor to the pathogenesis but not as being responsible for the development of the pathology. Probably more experience and family studies will allow to classify more clearly this variant.

Among the 44 patients harboring a class 3 variant, excepting p.(Tyr282Asp) variant, 14 patients harbored another variant. This represents about $2 \%$ of our cohort which is not negligible. This highlights the necessity for carrying on the investigations on this gene.

Unlike p.(Tyr282Asp), the pathogenicity of the variant p.(Tyr394Ser), localized in the CCID domain, is well admitted in literature (14). It has been shown that this variant is enriched in Ashkenazi Jewish kindreds with PHPT (16). It was identified in three index cases in our cohort. The ages of onset were 28,46 and 78 years.
The youngest was a male who presented with severe hypercalcemia linked to unique adenoma and family history of PHPT, the second one was a female with recurrent parathyroid hyperplasia and the third one was a male with PHPT, but no more clinical information was available. Thus, heterogeneity of clinical and anatomopathological presentations are observed with this variant. Incomplete penetrance associated with this GCM2 sequence variation was also highlighted by the family study presented in this article, with two siblings of more than 55 years old presenting with normalcalciumand phosphate levels. Our observation is in agreement with the recent article of Riccardi et al. (17) who studied GCM2 variants localized in CCID in sporadic PHPT and concluded that the penetrance of these variants was low. We thus classified this variant as a class 4 variant (likely pathogenic).

Notwithstanding the limits of our study due to lack of some biological and anatomopathological data, we suggest that GCM2 sequencing should be included in the systematic genetic screening of PHPT for patients with a familial history of PHPT or exhibiting a sporadic presentation before the age of 50 years or with recurrent/ persistent PHPT or with multiple gland parathyroid lesions. Next-generation sequencing facilitates this process (18). However, the interpretation of the sequence variations identified in GCM2 is quite difficult, due to the lack of functional studies available in routine diagnosis 
laboratory to determine the consequences of the variants, and the incomplete penetrance of likely pathogenic variants. This also highlights the importance of discussion between geneticists and clinicians in multidisciplinary concentration meetings and validation of variants by expert groups such as TENGEN in France, which achieves it for many years for MEN1 variants (19). So, in front of incomplete penetrance and variants of uncertain significance, what about management of patients harboring those variants? Follow-up of asymptomatic patients harboring such a variant is not excessively binding. They should just be monitored for calcemia and PTH to be able to detect the onset of the pathology, which can happen very late in life indeed never as the penetrance is incomplete.

To conclude, we propose to include this gene in panels for PHPT screening. The proposition of genetic screening for all patients of less than 50 years with PHPT appears to be still valid, as diagnosis of genetically determined PHPT may be younger for patients with HRPT2 or MEN1 mutation but older for patients with CASR or GCM2 mutation. Up till now, many GCM2 variants are classified as variants of uncertain significance. However, improvement in the future of our knowledge about this gene will help us in the tricky interpretation and classification of such variants.

\section{Declaration of interest}

The authors declare that there is no conflict of interest that could be perceived as prejudicing the impartiality of this study.

\section{Funding}

This work was supported by funding from $\mathrm{CHU}$ de Lille, Assistance Publique Hôpitaux de Marseille, Hospices Civils de Lyon, Assistance Publique Hôpitaux de Paris, France.

\section{Acknowledgments}

The authors thank all the patients and our clinical collaborators who referred their patients to us for molecular evaluation, and technicians for their expert technical help. This study was conducted on behalf of the TENGEN group.

\section{References}

1 El Lakis M, Nockel P, Gaitanidis A, Guan B, Agarwal S, Welch J, Simonds WF, Weinstein L, Marx S, Nilubol N et al. Probability of positive genetic testing results in patients with family history of primary hyperparathyroidism. Journal of the American College of Surgeons 2018226 933-938. (https://doi.org/10.1016/j. jamcollsurg.2018.01.007)

2 DeLellis RA \& Mangray S. Heritable forms of primary hyperparathyroidism: a current perspective. Histopathology 201872 117-132. (https://doi.org/10.1111/his.13306)
3 Marini F, Cianferotti L, Giusti F \& Brandi ML. Molecular genetics in primary hyperparathyroidism: the role of genetic tests in differential diagnosis, disease prevention strategy, and therapeutic planning. A 2017 update. Clinical Cases in Mineral and Bone Metabolism 201714 60-70. (https://doi.org/10.11138/ccmbm/2017.14.1.060)

4 D'Agruma L, Coco M, Guarnieri V, Battista C, Canaff L, Salcuni AS, Corbetta S, Cetani F, Minisola S, Chiodini I et al. Increased prevalence of the GCM2 polymorphism, Y282D, in primary hyperparathyroidism: analysis of three Italian cohorts. Journal of Clinical Endocrinology and Metabolism 201499 E2794-E2798. (https:// doi.org/10.1210/jc.2014-2857)

5 Guan B, Welch JM, Sapp JC, Ling H, Li Y, Johnston JJ, Kebebew E, Biesecker LG, Simonds WF, Marx SJ et al. GCM2-activating mutations in familial isolated hyperparathyroidism. American Journal of Human Genetics 201699 1034-1044. (https://doi.org/10.1016/j. ajhg.2016.08.018)

6 El Lakis M, Nockel P, Guan B, Agarwal S, Welch J, Simonds WF, Marx S, Li Y, Nilubol N, Patel D et al. Familial isolated primary hyperparathyroidism associated with germline GCM2 mutations is more aggressive and has a lesser rate of biochemical cure. Surgery 2018 163 31-34. (https://doi.org/10.1016/j.surg.2017.04.027)

7 Naveh-Many T \& Silver J. Transcription factors that determine parathyroid development power PTH expression. Kidney International 201893 7-9. (https://doi.org/10.1016/j.kint.2017.08.026)

8 Richards S, Aziz N, Bale S, Bick D, Das S, Gastier-Foster J, Grody WW, Hegde M, Lyon E, Spector E et al. ACMG Laboratory Quality Assurance Committee. Genetics in Medicine 201517 405-424. (https://doi.org/10.1038/gim.2015.30)

9 Amendola LM, Jarvik GP, Leo MC, McLaughlin HM, Akkari Y, Amaral MD, Berg JS, Biswas S, Bowling KM, Conlin LK et al. Performance of ACMG-AMP variant-interpretation guidelines among nine laboratories in the Clinical Sequencing Exploratory Research Consortium. American Journal of Human Genetics 201698 1067-1076. (https://doi.org/10.1016/j.ajhg.2016.03.024)

10 Castellano E, Attanasio R, Boriano A, Pellegrino M, Garino F, Gianotti L \& Borretta G. Sex difference in the clinical presentation of primary hyperparathyroidism: influence of menopausal status. Journal of Clinical Endocrinology and Metabolism 2017102 4148-4152. (https://doi.org/10.1210/jc.2017-01080)

11 Mizamtsidi M, Nastos C, Mastorakos G, Dina R, Vassiliou I, Gazouli M \& Palazzo F. Diagnosis, management, histology and genetics of sporadic primary hyperparathyroidism: old knowledge with new tricks. Endocrine Connections 20187 R56-R68. (https://doi. org/10.1530/EC-17-0283)

12 Yamada T, Tatsumi N, Anraku A, Suzuki H, Kamejima S, Uchiyama T, Ohkido I, Yokoo T \& Okabe M. Gcm2 regulates the maintenance of parathyroid cells in adult mice. PLoS ONE 201914 e0210662. (https://doi.org/10.1371/journal.pone.0210662)

13 Morito N, Yoh K, Usui T, Oishi H, Ojima M, Fujita A, Koshida R, Shawki HH, Hamada M, Muratani M et al. Transcription factor MafB may play an important role in secondary hyperparathyroidism. Kidney International 201893 54-68. (https://doi.org/10.1016/j. kint.2017.06.023)

14 Marx SJ \& Goltzman D. Evolution of our understanding of the hyperparathyroid syndromes: a historical perspective. Journal of Bone and Mineral Research 201934 22-37. (https://doi.org/10.1002/ jbmr.3650)

15 Marchiori E, Pelizzo MR, Herten M, Townsend DM, Rubello D \& Boschin IM. Specifying the molecular pattern of sporadic parathyroid tumorigenesis-the Y282D variant of the GCM2 gene. Biomedicine and Pharmacotherapy 201792 843-848. (https://doi.org/10.1016/j. biopha.2017.05.028)

16 Guan B, Welch JM, Vemulapalli M, Li Y, Ling H, Kebebew E, Simonds WF, Marx SJ \& Agarwal SK. Ethnicity of patients with germline GCM2-activating variants and primary 
hyperparathyroidism. Journal of the Endocrine Society 2017 1488-499. (https://doi.org/10.1210/js.2017-00043)

17 Riccardi A, Aspir T, Shen L, Kuo CL, Brown TC, Korah R, Murtha TD, Bellizzi J, Parham K, Carling T et al. Analysis of activating GCM2 sequence variants in sporadic parathyroid adenomas. Journal of Clinical Endocrinology and Metabolism 2019104 1948-1952. (https:// doi.org/10.1210/jc.2018-02517)

18 Persani L, de Filippis T, Colombo C \& Gentilini D. Genetics in endocrinology: genetic diagnosis of endocrine diseases by NGS: novel scenarios and unpredictable results and risks. European Journal of Endocrinology 2018179 R111-R123. (https://doi.org/10.1530/EJE18-0379)

19 Romanet P, Mohamed A, Giraud S, Odou MF, North MO, Pertuit M, Pasmant E, Coppin L, Guien C, Calender A et al. UMD-MEN1 database: an overview of the 370 MEN1 variants present in 1676 patients from the French population. Journal of Clinical Endocrinology and Metabolism 2019104 753-764. (https://doi.org/10.1210/ jc.2018-01170)

Received 16 August 2019

Revised version received 28 October 2019

Accepted 30 October 2019 